\title{
El enfoque del foco en la forma y las situaciones de movimiento en el aula de ASL: Actividades de interpretación y producción.
}

MERITXELl MuÑOZ CARRASCO

Universitat Autónoma de Barcelona

Meritxell.Munoz@uab.cat

Resumen: Las explicaciones que el aprendiente de español recibe ante la pregunta de por qué no se puede decir escalé arriba de la montaña, corrí adentro de la casa (de afuera hacia adentro) o gateé afuera de la habitación (de dentro a afuera) son que esos verbos no funcionan asi en español, que los nativos no lo dicen así o que no suena bien así. Todas ellas son explicaciones inoperativas, frustrantes y que no conducen a que el input llegue a convertirse en intake. En una primera parte teórica, este artículo describe cómo las lenguas expresan las situaciones de movimiento $\mathrm{y}$, en una segunda parte práctica, sugiere actividades, tanto de interpretación como de producción, de input estructurado desde el enfoque de foco en la forma para llevar estas estructuras lingüísticas al aula de ASL.

Palabras clave: foco en la forma, lingüística cognitiva, situaciones de movimiento.

Abstract: The explanations that the Spanish learner receives when asking why escalé arriba de la montaña, corrí adentro de la casa o gateé afuera de la habitación ${ }^{l}$ are inoperative, frustrating and do not help to the input to become intake. This article describes firstly, from a theoretical perspective how languages express motion events and some second language research, then, secondly, it suggests practical and structured input activities from the focus on form approach, comprehension and production, to take these linguistics structures into the Second Language Acquisition classroom.

Key words: focus on form, cognitive linguistics, motion events.

\footnotetext{
${ }^{1}$ Los ejemplos no se han traducido al inglés, esto es, I climbed up the mountain, I ran into the house, I crawled out the room porque, al ser una lengua de marco satélite, la traducción convierte las frases en gramaticales
} 


\section{Introducción}

El movimiento entendido como un cuerpo $\mathrm{u}$ objeto que se desplaza es un concepto universal presente en todas las lengua. Por ello, cada lengua cuenta con patrones lingüísticos para expresar desplazamientos o situaciones de movimiento tipo, Juan salió al jardín corriendo o María baila por la habitación. Adquirimos los patrones de pensar para hablar (Slobin, 2003) de nuestra L1, esto es, las pautas de conceptualización y de lexicalización, de forma innata e inconsciente. Por lo que cabe cuestionarse, ¿qué ocurre cuando aprendemos una L2 que es tipológicamente distinta a la L1? ¿Hemos de volver a adquirir esos patrones, es decir, hemos de repensar para hablar (Cadierno y Robinson, 2009) o son pautas que muestran resistencia a ser adquiridas?

Las numerosas investigaciones en ASL sobre las situaciones de movimiento en las que se ha estudiado si los aprendientes han adquirido las pautas lingüísticas de la L2 muestran que estos siguen trasladando los patrones lingüísticos de pensar para hablar de la L1 a la L2 y que no han asimilado a nivel nativo las estructuras lingüísticas. Teniendo en cuenta estos resultados y que las situaciones de movimiento no figuran en los manuales de $\mathrm{E} / \mathrm{LE}$, se debería empezar a plantear qué enfoques serían los más propicios y beneficiosos para los aprendientes y qué actividades favorecerían su aprendizaje.

En los tres siguientes apartados se explica de forma un poco más extensa cuáles son los componentes del movimiento, la tipología binaria de las lenguas de Talmy (2000) y explica de forma resumida los resultados de un estudio empírico sobre la adquisición de estos patrones. En el apartado quinto se presenta el foco en la forma como un enfoque posible para llamar la atención del estudiante a que realice la conexión entre la forma y el significado de estas pautas y se sugieren actividades de interpretación y de producción de input estructurado. En el último apartado se sugieren futuros estudios que aportarán luz sobre los beneficios de la inserción de estas estructuras en las clases de E/LE (extensibles a las clases de ASL de cualquier lengua materna y hacia cualquier lengua meta).

\section{El movimiento: Componentes y Tipología.}

El movimiento tiene cuatro componentes básicos: el Movimiento per se; la Figura como el objeto o persona que se mueve; el Camino o la dirección que toma la Figura en el movimiento y; la Base como lugar origen, lugar por el que pasa, o lugar final al que llega la Figura. A estos cuatro elementos internos, se añaden dos externos que son la Manera o forma en la que la Figura se mueve y, la Causa que provoca el movimiento. En los ejemplos (1) y (2) de Talmy (1985:60-61) aparecen los elementos descritos anteriormente:

$\begin{array}{rlll}\begin{array}{r}\text { (1) The pencil } \\ \text { Figura }\end{array} & \begin{array}{l}\text { rolled } \\ \text { Movimiento+Manera }\end{array} & \begin{array}{l}\text { off } \\ \text { Camino }\end{array} & \begin{array}{l}\text { the table } \\ \text { Base }\end{array} \\ \text { (2) The pencil } & \text { blew } & \text { off } & \text { the table } \\ \text { Figura } & \text { Movimiento+Causa } & \text { Camino } & \text { Base }\end{array}$


Talmy (2000), desde la Semántica Cognitiva, clasifica las lenguas en dos tipos: Lenguas de marco satélite y Lenguas de marco verbal. En las lenguas de marco satélite [LMS], el verbo principal expresa el Movimiento y la Manera o la Causa, mientras que el Camino se expresa en un constituyente externo al verbo principal llamado satélite. Talmy define satélite como:

determinados constituyentes presentes en muchas, si ni en todas las lenguas inmediatos a un verbo, que no son flexibles, auxiliares o argumentos nominales. Los satélites se relacionan con la raíz verbal como modificadores a un núcleo. Una raíz verbal junto con sus satélites forma un constituyente por derecho propio: un verbo complejo. ${ }^{2}$

Talmy (1985:102)

Las siguientes frases (Cadierno, 2008b: 263) muestran la estructura básica de las lenguas de marco satélite, donde el verbo principal (ran, løb) expresa el movimiento y la Manera, y los satélites (out, ud) expresan el Camino.

(3) The man ran out of the house

(4) Manden løb ud of huset

Otra particularidad de esta tipología es que pueden amalgamar más de un componente de Camino con un solo verbo principal (5), característica inexistente en las lenguas de marco verbal, las cuales necesitan un verbo de Camino que introduzca cada nueva trayectoria (6). Ejemplos extraídos de Slobin (2004:238):

(5) The frog crawled out of the jar and through the window into the woods

(6) La rana se escapó del frasco, salió por la ventana y se internó en el bosque

Las lenguas que pertenecen a la tipología de marco satélite son: las lenguas germánicas (el danés, el holandés, el inglés, el alemán, el sueco y el islandés), las lenguas eslavas (el checo, el polaco, el ruso, el serbocroata y el ucraniano), el finlandés, el húngaro y el chino mandarín entre otras.

En las lenguas de marco verbal [LMV] el verbo principal expresa el Camino y los componentes de Manera o Causa se expresan, en caso de ser necesarios para el contexto, en un constituyente externo al verbo, tales como, sintagma preposicional, gerundio, sintagma adverbial o adjetivo. Las lenguas románicas (el español, el portugués, el francés, el catalán, el rumano, el italiano y el gallego), las lenguas semíticas (el hebreo y el árabe marroquí), el turco, el vasco, el japonés, el groenlandés, el griego y el coreano entre otras pertenecen a esta tipología. Ejemplos de esta tipología en español (7) y en catalán (8) son (Cadierno, 2008b:264):

(7) El hombre salió de la casa corriendo

(8) L'home va sortir de la casa corrent

Las lenguas de marco verbal presentan un sistema dual de fusión (Aske, 1989; Slobin y

\footnotetext{
${ }^{2}$ Las citas han sido traducidas por la autora del artículo.
} 
Hoiting, 1994) según si la situación de movimiento implica un cruce de frontera [+CF], esto es, el punto origen y el punto final del movimiento son distintos, o no implica un cruce de frontera [-CF], esto es, el punto origen y el punto final son el mismo.

Cuando la situación de movimiento conlleva un $[+\mathrm{CF}]$ las lenguas de marco verbal se comportan siguiendo los parámetros prototípicos anteriormente descritos (9), es decir, el verbo principal describe el Camino y la Manera queda relegada, en caso de ser destacada y necesaria para el contexto, a un constituyente externo. En cambio, cuando la situación de movimiento no conlleva un [-CF], las lenguas de marco verbal usan los mismas pautas lingüísticas que las lenguas de marco satélite $(10,11,12)$, esto es, el verbo principal amalgama Movimiento y Manera.

(9) El hombre entró en la casa corriendo

(10) El hombre corrió hasta la casa

(11) The man ran up to the house

(12) The man ran into the house

De la explicación anterior, se deduce que todas las lenguas se clasifican, o bien como lenguas de marco verbal, o bien como marco satélite ${ }^{3}$, por lo que los estudiantes necesitan saber si la lengua que están adquiriendo se comporta como su propia lengua materna o si se comporta siguiendo otras pautas de lexicalización y, por consiguiente, de conceptualización. Esta idea enlaza con la hipótesis de pensar para hablar de Slobin (2003) expuesta en el apartado siguiente.

\section{La hipótesis de thinking for speaking o pensar para hablar de Slobin (2003, 2004)}

Esta hipótesis de pensar para hablar está en consonancia con la teoría whorfiana débil por la que la interpretación de la realidad difiere dependiendo de la lengua que uno hable. El hablante va a atender a aquellos rasgos de una situación dada que sean fácilmente codificables lingüísticamente en su propia lengua materna. Un hablante de español como L1 dará prioridad al Camino frente a la Manera, mientras que un hablante de inglés como L1 dará prioridad a la expresión detallada de la Manera mientras que dejará que otros componentes se infieran por el contexto, como las descripciones estáticas.

Si cada hablante describe o presta atención a aquellos componentes del movimiento que son destacados en su lengua materna y los describe utilizando las herramientas disponibles en su lengua, cabe preguntarse si ¿el aprendiente de español aprende la estructura conceptual que subyace a la estructura sintáctica y semántica? si ¿aprenderá qué componentes del movimiento son los destacados y qué componentes se relegan a un papel secundario? Esto es, el aprendiente hará el ejercicio de volver a pensar para hablar o "repensar" para hablar en su L2 (Cadierno y Robinson, 2009), o por el contrario, se servirá de los patrones de su L1 como punto de partida para describir situaciones de

\footnotetext{
${ }^{3}$ Hay lenguas que no encajan ni en una ni en otra clasificación. Para más información consúltese, entre otros: Slobin, D. I. (2004). The many ways to search for a frog: Linguistics typology and the expression of motion events. En S. Stromqvist y L.Verhoeven (Eds.), Relating events in narrative: Typological and contextual perspectives, (pp.219-257). Hillsdale, NJ: Lawrence Erlbaum.
} 
movimiento en su L2.

A fin de contestar las preguntas anteriormente formuladas, se han llevado a cabo numerosos estudios empíricos en ASL comparando lenguas de igual tipología y de distinta tipología. ${ }^{4}$ En el apartado siguiente se explica el estudio empírico realizado por Muñoz Carrasco (2015) entre aprendientes de español en tres estadios de aprendizaje distintos (A2, B1, B2) hablantes de inglés como L1 con los objetivos de: (1) investigar si los aprendientes recurren a su L1 como punto de partida; y, (2) verificar si la exposición a un input comprensible de la L2 es suficiente para la adquisición de la conceptualización y la lexicalización de estas estructuras.

\section{Este estudio}

El estudio experimental que aquí se presenta se llevó a cabo entre 2013-2015 con un total de 75 informantes. Estos informantes se dividían en 5 grupos: diez hablantes de español como L1 sin conocimientos de inglés; once hablantes de inglés como L1 sin conocimientos de español; 22 aprendientes de español L2 en un nivel A2; 19 aprendientes de español L2 en un nivel B1; y, 13 aprendientes de español en un nivel B2. Todos los aprendientes de español eran estudiantes universitarios de Estados Unidos que participaban en el programa Study Abroad que ofrece la Universitat Autònoma de Barcelona, con una estancia de cuatro meses en la que estudian asignaturas relacionadas con sus carreras y un curso de español de 90 horas.

Una de las preguntas de investigación motivo del estudio fue si los aprendientes han adquirido las estructuras lingüísticas para describir las situaciones de movimiento a nivel nativo o si en cambio recurren a la L1 como punto de partida.

La tarea que llevaron a cabo fue ver y describir por escrito en su lengua materna ${ }^{5} 14$ fragmentos de vídeos de Mr. Bean ${ }^{6}$. Siete de los fragmentos hacían referencia a una situación de movimiento sin cruce de frontera, tipo $\mathrm{Mr}$ Bean corre por la calle a pata coja, Mr. Bean baja unas escaleras; y, siete de los fragmentos hacían referencia a una situación de movimiento con cruce de frontera tipo Mr. Bean sale de su casa corriendo, Mr. Bean entra en un centro comercial.

La hipótesis de partida predecía que la influencia de la L1 sobre la L2 sería mayor en los aprendientes con un nivel de competencia lingüística inferior y que sería menor en los aprendientes con un nivel de competencia lingüística superior. Se esperaba que los aprendientes de español expresaran de la misma forma las oraciones $+\mathrm{CF}$ y $-\mathrm{CF}$ teniendo en cuenta que no hay distinción, si hay o no cruce de frontera, en su L1, esto es, que se esperaba que utilizaran indistintamente Caminé afuera de la casa para expresar tanto la idea de que la persona camina fuera de la casa porque se encuentra en el exterior, como la idea de que la persona sale de la casa caminando. Se esperaba que los aprendientes no expresasen el componente de Manera en sintagmas externos al

\footnotetext{
${ }^{4}$ En Muñoz Carrasco (2015) se puede encontrar un exhaustivo listado de los estudios de ASL sobre situaciones de movimiento entre lenguas de igual tipología y lenguas de diferente tipología.

${ }^{5}$ En este artículo se expone de forma muy sucinta y resumida el estudio experimental. La investigación fue más compleja puesto que se estudió la posible transferencia lingüística bidireccional entre la lengua materna y la segunda lengua.

${ }^{6}$ Los vídeos fueron seleccionados, editados y modificados por la autora de la investigación. Se pueden encontrar en el siguiente enlace http://youtu.be/75VBp7igCF4
} 
verbo con tanta frecuencia como el grupo de español L1 porque no es una pauta propia de su L1. De la misma forma, se predecía la aparición de "satelizaciones», esto es, palabras usadas simulando los satélites de Camino, como El perro saltó fuera de la ventana (Cadierno y Ruiz, 2006) donde fuera actúa como el satélite out en inglés.

Aunque los resultados muestran que los grupos de aprendientes expresan el componente de Manera en el verbo principal con mayor frecuencia, no es un dato estadísticamente significativo, posiblemente, debido a que los informantes no eran muy numerosos. También se observa que los grupos de aprendientes expresan la Manera en sintagmas externos al verbo significativamente menos que el grupo español L1. En referencia al Camino, queda confirmada la hipótesis de partida que advertía que los aprendientes mencionarían verbos de Camino con una frecuencia menor que el grupo español nativo, siguiendo los patrones de su L1. De la misma manera, se confirma la hipótesis que predecía la aparición de «satelizaciones» frases en las que apareciera una palabra simulando un satélite de Camino (detrás, fuera y abajo). Si bien son muy interesantes por ser traslaciones de pautas de la L1 a la L2, no fueron significativas desde el punto de vista estadístico:

(1) Caminó detrás de un túnel (A2)

(2) Está manejando afuera del aparcamiento (B1)

(3) Caminó abajo muchas escaleras (B2)

Aunque nuevamente no son estadísticamente significativos, aparecen casos de Trayectorias Múltiples en los grupos de aprendientes de español, mientras que no se observa ni una sola mención en el grupo de español nativo:

(4) La pelota de golf fue afuera del campo, arriba una valla y en un autobús ${ }^{7}$.

(5) La pelota de golf fue debajo de la valla y adentro el autobús.

Se observa que los grupos de aprendientes no han adquirido las restricciones semánticas que conllevan las situaciones de cruce de frontera, $y$, en consecuencia, la diferencia de patrones lingüísticos existentes en la L2. Los grupos de aprendientes usan el mismo patrón lingüístico (Verbo Manera + Camino en un satélite) heredado de su L1 para expresar situaciones de $+\mathrm{CF}$ y $-\mathrm{CF}$. En los siguientes ejemplos los aprendientes usan las estructuras de $-\mathrm{CF}$ de lenguas de marco verbal para describir situaciones de $+\mathrm{CF}$ :

(6) Caminó a la tienda (vídeo 14 donde Mr. Bean entra en una tienda, A2)

(7) Camina adentro de un túnel (vídeo 10 donde Mr. Bean se mete en un túnel, B1)

(8) Maneja un carro amarillo afuera de un garaje (vídeo 2 donde Mr. Bean sale de un garaje en un coche amarillo, B2)

Los resultados muestran que no se han adquirido las pautas lingüísticas a nivel nativo ni en el caso de los aprendientes de mayor competencia lingüística. La hipótesis de entrada que predecía que cuanta mayor fuera la competencia del estudiante, menor sería la

\footnotetext{
${ }^{7}$ Estas frases se refieren al vídeo número 6 en el que se ve como Mr Bean golpea una pelota de golf, ésta salta por encima de una valla, rebota por la zona de golf, por un banco y entra en un autobús.
} 
influencia de la L1 sobre la L2 no queda confirmada a la luz de los resultados. No hay diferencias significativas en aquellas variables dependientes que son transferencia directa de la L1 a la L2, esto es, rasgos diferentes entre los grupos de aprendientes y el grupo nativo español. Estas variables dependientes son el menor uso de verbos de Camino y el menor uso de la descripción de la Manera en sintagmas independientes. Además de estas dos variables significativas desde el punto de vista estadístico, se han encontrado ejemplos muy interesantes de «satelizaciones» y de Trayectorias Múltiples, muestra de pautas transferidas de L1 a L2.

Si los aprendientes expuestos a un input comprensible no dan muestras de haber interiorizado las pautas lingüísticas subyacentes a las situaciones de movimiento, cabe pensar que esa exposición no es suficiente y que, por consiguiente, el aprendiente se podría beneficiar de una metodología que dirigiera su atención hacia estos patrones y que le ayudara a hacer la conexión adecuada entre el significado y la forma. En el próximo apartado se propone el foco en la forma como posible método didáctico.

\section{Focus on form e Instrucción de Procesamiento}

Por una parte, los resultados extraídos del estudio anterior muestran que el enfoque de atención al significado, esto es, sólo exposición a un input comprensible, no es suficiente para que los estudiantes adquieran los patrones de lexicalización de las situaciones de movimiento de su L2, especialmente aquellos que difieren de su L1, por ser lenguas de tipología diferente.

Por otra parte, estas estructuras no se contemplan, hasta donde yo sé, en ningún libro de texto, temario, ni siquiera están recogidas en el Plan Curricular Cervantes más allá de aparecer los verbos de movimiento inventariados en las secciones de Nociones Específicas de cada nivel de competencia lingüística

Por todo ello, parece conveniente pensar que un enfoque dirigido a la forma, sin obviar el significado, podría ser beneficioso para llevar estas estructuras al aula de E/LE y de ASL en general. Un buen punto de partida podría ser el enfoque de atención a la forma o focus on form. Éste fue planteado por Long (1991) como contrapropuesta al enfoque imperante anterior, focus on meaning o atención al significado, como método que dirige la atención del aprendiente hacia la forma gramatical conectada con el significado. Concretamente, dentro del enfoque de atención a la forma, adoptaremos la instrucción de procesamiento (Van Patten, 1996) como forma de operativizar esa conexión entre significado y forma para llevar estas estructuras al aula de E/LE.

Esta metodología, presente en el ámbito de la investigación desde principios de los años 90, da a los aprendientes la oportunidad de procesar formas meta por medio de actividades con un input que ha sido especialmente estructurado para aumentar las posibilidades de desarrollo de la interlengua y una eventual adquisición

Llopis García, 2015:8

Esta metodología se relaciona con la Lingüística Cognitiva en tanto que las formas lingüísticas están a merced de la intención comunicativa del hablante y del uso del lenguaje. La instrucción de procesamiento trata de hacer conexiones entre la forma lingüística y el significado sobre estructuras que, de otra manera, pasarían desapercibidas para el estudiante. Como aconseja Llopis García (2015:13) «se proponen 
los enfoques cognitivo-operativos para la presentación explícita de formas gramaticales en el aula de E/LE, y el foco en la forma como metodología para su práctica.»

La estructura de una unidad didáctica desde la perspectiva del procesamiento de input es (Cadierno, 2008b):

1. Explicación explícita de las estructuras lingüísticas ligada a su significado para que el estudiante sea consciente del propósito comunicativo que implican.

2. Explicación sobre las estrategias de procesamiento que inducen a la comprensión errónea del input, esto es, meta reflexión sobre cómo su L1 expresa estas frases.

3. Actividades de input/output estructurado y comprensible diseñadas para que el estudiante conecte el significado con la estructura lingüística.

Para explicar de forma explícita las estructuras lingüísticas, se pueden mostrar diferentes vídeos en los que se ven situaciones de movimiento sin cruce de frontera acompañados de la frase que los describe. Se reflexiona sobre el significado, sobre el elemento que describe el Camino, el elemento que describe la Manera, y se pide al estudiante que piense cómo se describen estas acciones en su lengua materna. Para hacer un mayor hincapié entre la forma y el significado, se pueden establecer unas marcas visuales, tales como escribir en rojo el componente de Manera y, en azul, el componente de Camino.

Una vez, explicadas las situaciones de movimiento sin cruce de frontera, se muestran diferentes vídeos en los que se ven situaciones de movimiento con cruce de frontera acompañadas de las frases que las describen. Se reflexiona sobre el significado, sobre el elemento que describe el Camino, el elemento que describe la Manera y se le pide al estudiante que piense cómo se describen esas acciones en su lengua materna.

Una vez explicado el mecanismo de estas estructuras y hecho una reflexión sobre la expresión de las mismas en su lengua materna, se procede a que el estudiante las practique con actividades de interpretación o de input estructurado y con actividades de producción o output estructurado.

A continuación se presenta un cuadro con ejemplos de ambas actividades. En la parte de la izquierda se sugieren actividades de input estructurado y, en la parte de la derecha, se proponen actividades homónimas pero de output estructurado. De la misma forma, en un primer lugar hay actividades centradas en el significado del léxico antes de tratar aspectos gramaticales; en un segundo lugar, destacan actividades en las que estas pautas están insertas en oraciones; $y$, en un tercer lugar, se llega a actividades en las que las situaciones de movimiento aparecen en un texto ${ }^{8}$.

\footnotetext{
8 En el siguiente enlace se puede ver la presentación que se mostró en el XIII Foro de profesores de Español como Lengua Extranjera (FOROELE) de Valencia sobre actividades de producción y de interpretación para trabajar las situaciones de movimiento en el aula. Se pueden encontrar ejemplos de algunas de las actividades arriba propuestas.

https://uab.academia.edu/MeritxellMu\%C3\%B1ozCarrasco
} 


\begin{tabular}{|c|c|}
\hline $\begin{array}{l}\text { INTERPRETACIÓN } \\
\text { actividades de input estructurado }\end{array}$ & $\begin{array}{l}\text { PRODUCCIÓN } \\
\text { actividades de output estructurado }\end{array}$ \\
\hline $\begin{array}{l}\text { Relacionar léxico de Manera (correr, saltar) y } \\
\text { Camino (ir, volver) con dibujos. Flashcards. }\end{array}$ & $\begin{array}{l}\text { Producir el léxico de Manera y Camino a partir de } \\
\text { los dibujos. Flashcards. }\end{array}$ \\
\hline $\begin{array}{l}\text { Clasificar una lista de palabras según expresen } \\
\text { Manera o Camino (a la pata coja, ascender, } \\
\text { bailar...) }\end{array}$ & $\begin{array}{l}\text { Escribir más muestras de palabras que expresen } \\
\text { Manera o Camino }\end{array}$ \\
\hline Clasificar frases según sean $+\mathrm{CF}$ o $-\mathrm{CF}$ & $\begin{array}{l}\text { Escribir otros ejemplos }+ \text { CF o -CF a partir de un } \\
\text { modelo }\end{array}$ \\
\hline $\begin{array}{l}\text { En parejas relacionan preguntas con respuestas: } \\
\text { a.¿Cómo sales de casa cuando llegas tarde? } \\
\text { b.¿Cómo entrarías a clase si te tocara la lotería? } \\
\text { c. ¿Cómo te escaparías de una casa que está en un } \\
\text { árbol? } \\
\text { 1.Salgo de casa corriendo } \\
\text { 2. Entraría bailando } \\
\text { 3. Me escaparía bajando con una cuerda }\end{array}$ & $\begin{array}{l}\text { En parejas se hacen preguntas sin opciones para } \\
\text { relacionar, } \\
\text { a.¿Cómo sales de casa cuando llegas tarde? } \\
\text { b.¿Cómo entrarías a clase si te tocara la lotería? } \\
\text { c.¿Cómo te escaparías de una casa que está en un } \\
\text { árbol? }\end{array}$ \\
\hline $\begin{array}{l}\text { Visionado de un video y relacionar con la frase } \\
\text { correcta: } \\
\text { (Mr. Bean sale de un centro comercial corriendo) } \\
\text { a. Mr. Bean corre por el centro comercial } \\
\text { b. Mr. Bean sale corriendo del centro } \\
\text { comercial }\end{array}$ & $\begin{array}{l}\text { Visionado de un video y describir lo que ven de } \\
\text { forma oral o escrita, en parejas, incluso un } \\
\text { miembro de la pareja ve el vídeo y el otro no, o en } \\
\text { grupos. }\end{array}$ \\
\hline $\begin{array}{l}\text { Con tarjetas, interpretar la acción por la clase } \\
\text { a. Salta a la pata coja hasta la pizarra } \\
\text { b. Sal de clase bailando }\end{array}$ & $\begin{array}{l}\text { Con tarjetas, un estudiante interpreta una acción y } \\
\text { el resto ha de adivinar qué hace. }\end{array}$ \\
\hline $\begin{array}{l}\text { Dictapictionary: Se lee una frase y han de dibujar } \\
\text { la acción que describe }\end{array}$ & $\begin{array}{l}\text { Dictapictionary: Ellos mismos crean las frases } \\
\text { para que el otro grupo las dibuje. }\end{array}$ \\
\hline $\begin{array}{l}\text { Yincana por el centro docente siguiendo las } \\
\text { instrucciones dadas por el profesor. } \\
\text { a.Entra en la oficina a la pata coja }\end{array}$ & $\begin{array}{l}\text { Yincana por el centro docente siguiendo las } \\
\text { instrucciones previamente creadas por otro grupo. }\end{array}$ \\
\hline $\begin{array}{l}\text { Comprensión auditiva } \\
\begin{array}{l}\text { a. relaciona con la respuesta correcta } \\
\text { b. ordena la historia según el audio }\end{array}\end{array}$ & $\begin{array}{l}\text { Comprensión auditiva } \\
\begin{array}{l}\text { a. inventa el final de la historia } \\
\text { b. contesta preguntas sobre el audio, sin }\end{array}\end{array}$ \\
\hline
\end{tabular}




\begin{tabular}{|c|l|}
\hline & \multicolumn{1}{|c|}{ darle posibles respuestas } \\
\hline Comprensión lectora & Comprensión lectora \\
a. Identificar las palabras que expresan la & $\begin{array}{l}\text { Manera y el Camino en un texto } \\
\text { misma historia que después han de resumir al } \\
\text { compañero/a. }\end{array}$ \\
b. $\begin{array}{l}\text { Texto tradicional y preguntas con } \\
\text { opciones }\end{array}$ & b. Preguntas sobre el texto sin opciones \\
c. $\begin{array}{l}\text { Ordenar las frases acorde con lo visto en } \\
\text { un video (corto, dibujos animados) }\end{array}$ & \\
\hline
\end{tabular}

Tabla 1 Actividades de producción e interpretación

\section{Futuros estudios}

En este artículo se ha propuesto la aplicación del enfoque de foco en la forma desde los conceptos teóricos de la Lingüística Cognitiva para el aprendizaje de las situaciones de movimiento. Asimismo, las situaciones de movimiento se podrían llevar al aula desde otros enfoques didácticos, tales como el enfoque léxico o el enfoque comunicativo, que podrían dar resultados muy positivos. Son necesarios estudios experimentales sobre qué enfoques y qué tipo de actividades ayudan más a los aprendientes a adquirir estas estructuras lingüísticas hasta ahora ignoradas en las clases de E/LE.

\section{Bibliografía}

Aske, J. (1989). «Path predicates in English and Spanish: A closer look». En Proceedings of the 15th Annual Meeting of the Berkeley Linguistics Society. Berkeley, CA: Berkeley Linguistics Society, 1-14.

CAdierno López, T. (2008b). «Motion events in Danish and Spanish: A focus on form pedagogical approach». En De Knop, S. \& De Rycker, T. (ed.); Cognitive Approaches to Pedagogical Grammar (pp.259-294). Berlín/Nueva York: Mouton de Gruyter.

CAdierno LóPez, T., y Ruiz, L. (2006). «Motion events in Spanish L2 acquisition». En Annual Review of Cognitive Linguistics, 4, 183-216

CADIERno LóEz, T., y Robinson, P. (2009). «Language typology, task complexity and the development of L2 lexicalization patterns for describing motion events». En Annual Review of Cognitive Linguistics, 7, 245-276.

Llopis GARCÍA, R. (2015). «La instrucción gramatical en la Adquisición de Segundas Lenguas -revisión de ayer para propuestas de hoy». En RedELE, número 16, 1-15.

LONG, M. (1991). «Focus on form: A design feature in language teaching methodology. En K. De Bot, R. Ginsberg, \& C. Kramsch (ed.); Foreign language research in crosscultural perspective (pp. 39-52). Amsterdam: John Benjamins.

MuÑoz CARRASCO, M. (2015). La transferencia bidireccional inglés-español en las situaciones de movimiento. Tesis doctoral. No publicada. Madrid: Universidad Antonio de Nebrija. 
SlobIN, D. I. (2003) «Language and thought online: Cognitive consequences of linguistic relativity». En D. Gentner y S. Goldin-Meadow (ed.); Language in mind: Advances in the study of language and thought. (pp.157-192). Cambridge, MA: MIT Press.

SLoBIN, D. I. (2004). «The many ways to search for a frog: Linguistics typology and the expression of motion events». En S. Stromqvist y, L.Verhoeven (ed.); Relating events in narrative: Typological and contextual perspectives (pp.219-257). Hillsdale, NJ: Lawrence Erlbaum.

Slobin, D. I., y Hoiting, N. (1994). «Reference to movement in spoken and signed language: Typological considerations» En Proceedings of the 20th Annual Meeting of the Berkeley Linguistic Society (pp 487-505). Berkeley: Berkeley Linguistics Society.

TALMY, L. (1985). «Lexicalizations patterns: Semantic structure in lexical forms» En T. Shopen (ed.), Language typology and syntactic description (pp. 39-149). Cambridge: Cambridge University Press.

TALmy, L. (2000b). Toward a cognitive semantics, volume 2: Typology and Process in concept structuring. Cambridge: MIT Press.

VanPatten, B. 1996. Input Processing and Grammar Instruction: Theory and Research. Nueva Jersey: Ablex Publishing Corporation. 